\title{
VIBRATION ANALYSIS OF A FLAT PLATE UNDER AERODYNAMIC LOADING
}

\author{
M. Farag ${ }^{1}$, A. El-Badawy ${ }^{1}$, T. Nasreldeen ${ }^{1}$ and R. Mohamed ${ }^{1}$ and M. Shawki ${ }^{2}$, \\ ${ }^{1}$ Department of Mechanical Engineering, Faculty of Engineering, Al-Azhar University \\ ${ }^{2}$ Department of Mechatronics Engineering, Higher Technological Institute
}

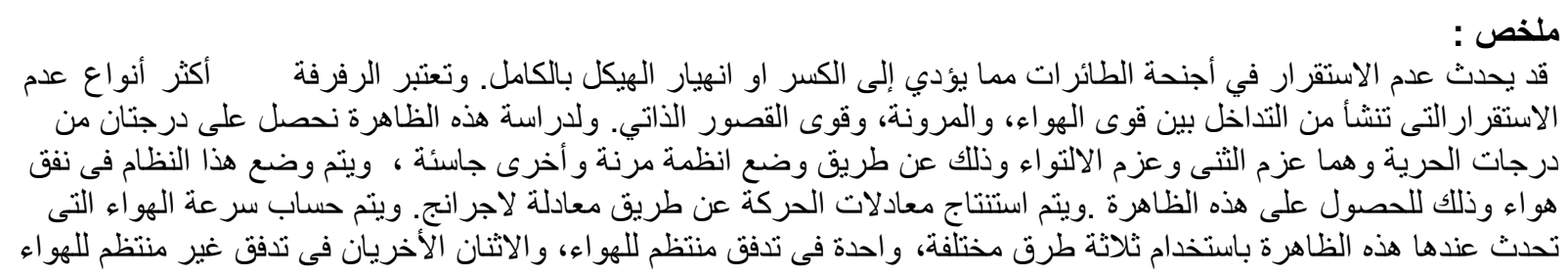

\begin{abstract}
Aeroelastic instabilities may occur in aircraft surfaces, leading to fatigue or structure failure. Flutter is an aeroelastic instability that results in a self-excited divergent oscillatory behavior of the structure. A classical two-degree-of-freedom flutter is a combination of bending and torsion vibration modes. A flexible mount system has been developed for flutter tests with rigid wings in wind tunnel. This flexible mount has to provide a well-defined two-degrees-of-freedom system on which rigid wings encounter flutter. Experi-mental Modal Analysis (EMA) and Finite Element Model Analysis (FEM) were performed to verify the natural frequencies and modes prior to any wind tunnel flutter test. The equations of motion of the system were developed using Lagrange's equation. The critical flutter speed was determined by three different methods: the $\boldsymbol{p}$-method for steady flow, the classical flutter analysis and the $\boldsymbol{k}$-method for unsteady flow, and comparing with experimental results.
\end{abstract}

\section{KEYWORDS: Aeroelasticity, flutter, flexible Structures, Wind Tunnel Tests, Experimental Modal Analysis, Finite Element Model Analysis.}

\section{INTRODUCTION}

Aeroelasticity is the term used to denote the field of study concerned with the interaction between the deformation of an elastic structure in an airstream and the resulting aerodynamic force. There are two main fields in the study of aeroelasticity. First, static aeroelasticity deals with the interaction between elastic and aerodynamic forces neglecting the effects of inertial forces (in such a way the inertial forces have little effect). Any deformation that occurs on a lifting surface causing it to deflect can result in increasing the applied load which may lead to the failure of the surface which is known as divergence. Second, dynamic aeroelasticity deals with the interaction between elastic, aerodynamic and inertial forces [1].

Aeroelastic divergence and flutter is often the most encountered phenomena that cause structural failure. It is a self-feeding destructive vibration where the lifting surface extracts the air stream energy causing it to oscillate resulting in an unstable condition. It occurs when the aerodynamic load is increased due to the vibrational movement of the object causing the object to move further. During the period of aerodynamic excitation, if the energy is larger than the system's 
natural damping, the vibration's level will increase, resulting in self-exciting oscillation. The vibration levels can only be limited when the system's aero-dynamic or mechanical damping equals the energy input, which often results in large amplitudes leading to quick failure. Therefore, in order to avoid flutter, any structure exposed to aerodynamic forces should be designed carefully within known parameters. The collapse of the original Tacoma Narrows Bridge is one of the famous examples of flutter phenomenon.

Many authors through the years have studied flutter which applied to aeroelastic models [2], [3]. These models include parts of aircrafts, like rigid or flexible wing models, or a complete flexible model of an aircraft.

During 1970s, the first demonstration of experimental flutter at the NASA Langley Research Centre was performed in its Transonic Dynamics Tunnel. A cantilevered delta wing with leading and trailing edges flaps as actuators was then tested based on the aero- dynamic energy concept [4]. The B-52, YF-17, X-29, and DAST (Drone for Aerodynamic and Structural Tests) has made significant contributions to a better understanding of aeroelastic phenomenon[3].

During the 1980s the active flexible wing (AFW) was developed. The concept of this programme consisted of the design, wind tunnel test, and evaluation of Three Active Flutter Suppression Controllers. Classic and modern control techniques were exploited with this experimental model [5],[6].

In the 1990s, the Benchmark Active Control Technology was developed. Wind tunnel experiments were performed in order to obtain data to validate computational fluid dynamics codes, to verify aeroservoelastic design and analysis tools, and to provide an active control testbed. Classical, modern, and non-conventional controllers were tested with this system [7],[8]. In the same period, experimental non-linear aeroelastic control studies using adaptive control schemes were performed [9]. Historical perspectives in non-linear aeroelasticity developments are presented in reference [10].

During the 2005s, a flexible mount system had been developed for flutter tests with rigid wings in wind tunnels. Experimental Modal Analysis (EMA) are done before wind tunnel tests to verify the dynamical characteristics of the system [11].

In the 2014s, a multi-input/multi-output (MIMO) time-delay feedback controller was designed to actively suppress the flutter instability of a multiple-actuated-wing (MAW) wind tunnel model in the low subsonic flow regime [12].

During the 2015s, a delayed controller was designed for active flutter suppression of a three dimensional wing model. The delayed controller was digitally implemented and tested for the three-dimensional wing model in NH-2 subsonic wind-tunnel [13].

In this work, wind tunnel tests were performed with the physical system to achieve flutter state parameters. Pitch and plunge responses were measured in time domain in order to verify the critical flutter speed and comparing with theoretical three different methods: the $\boldsymbol{p}$-method for steady flow, the classical flutter analysis and the $\boldsymbol{k}$-method for unsteady flow. The system was tested from low wind tunnel velocities up to velocities as near as possible of flutter velocity.

Experimental Modal Analysis (EMA) and Finite Element Analysis (FEA) were performed to verify the natural frequencies and modes prior to any wind tunnel flutter test. The equations of motion were developed using Lagrange's equations and the Principle of Virtual Work.

\section{FLUTTER MOUNT SYSTEM}

The flexible mount system provides a well-defined two-degree-of-freedom dynamic system on which a rigid wing will encounter classical flutter. A side and perspective view of the flutter mount system are presented in Figure 1.

The flutter mount system consists of a moving plate supported by a system of four circular rods and a centered flat-plate strut. These rods and the flat-plate provide the elastic constraints and the rigid wing model fixed in the moving plate will oscillate in a two-degree-of-freedom mode, pitch and plunge, when flutter is encountered.

The rods are made of stainless steel, flat-plate strut, and moving plate are made of steel and all connections are fixed- fixed end. Their dimensions are: rods diameter, $0.0030 \mathrm{~m}$; moving plate, $(600 \times 300) \mathrm{mm}$, flat plate strut, $(550 \times 10 \times 2) \mathrm{mm}$, wing model, a thin airfoil with (500x 300) $\mathrm{mm}$. 


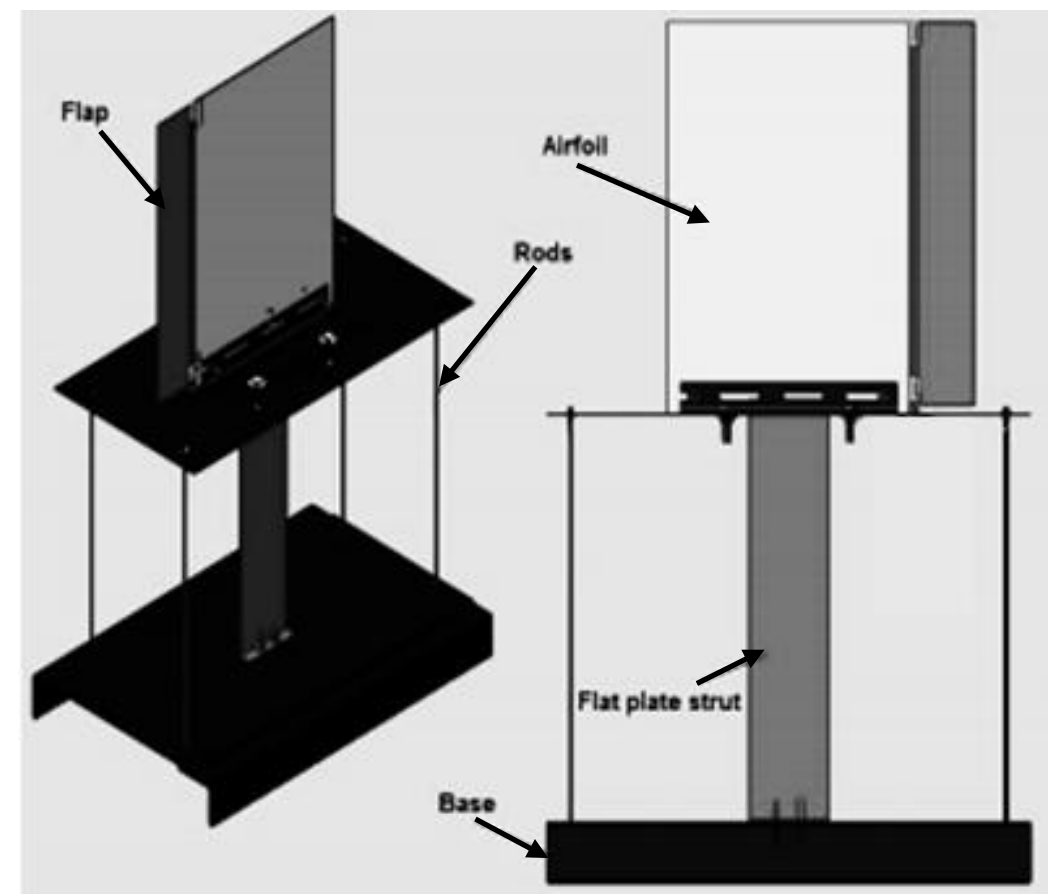

Fig. 1. Side and perspective views of the divergence and flutter mount system.

The wind-off characteristics of the flutter mount system are strongly determined by the dimensions of the flat-plate strut, the rods, and the mass of the moving plate and wing model. Modifications in the length and cross-section of the flat-plate strut and rods modify the frequencies and mode shapes of the flexible mount system. However, different wing models can be tested with the same flexible mount system by adding weights to the after and before inboard position in the moving plate, modifying the mass and inertia of the system.

These weights can also be used to decouple the pitch and plunge modes by moving the center of gravity of the flexible mount and wing model to the system elastic axis. The system elastic axis is located in the vertical center-line of the flat-plate strut and center of the moving plate. The four rods assure a parallel pitch and plunge displacement relative to the wind tunnel wall.

\section{WIND-OFF DYNAMIC CHARACTERISTICS}

An experimental modal analysis (EMA) and finite element model analysis (FEM) were performed to verify the natural frequencies and modes prior to wind tunnel flutter test. The measurement points are located at the flat-plate strut because it provides the elastic constraints to the system, the wing model being considered rigid.

First, to calculate natural frequencies using experimental modal analysis (EMA).The exciter employed during the tests is an impact hammer ENDCVOO model 2302-X. The outputs are measured by an accelerometer ISA-RP-37.2. This accelerometer is a capacitive one with a frequency range beginning at low values, being able to measure the low frequencies expected for this system. A dual-channel Bruel and Kjaer Dynamic Signal Analyzer type 3560C is used to obtain frequency response functions. Figure 2 showing that Schematic of hardware installation on structure. 


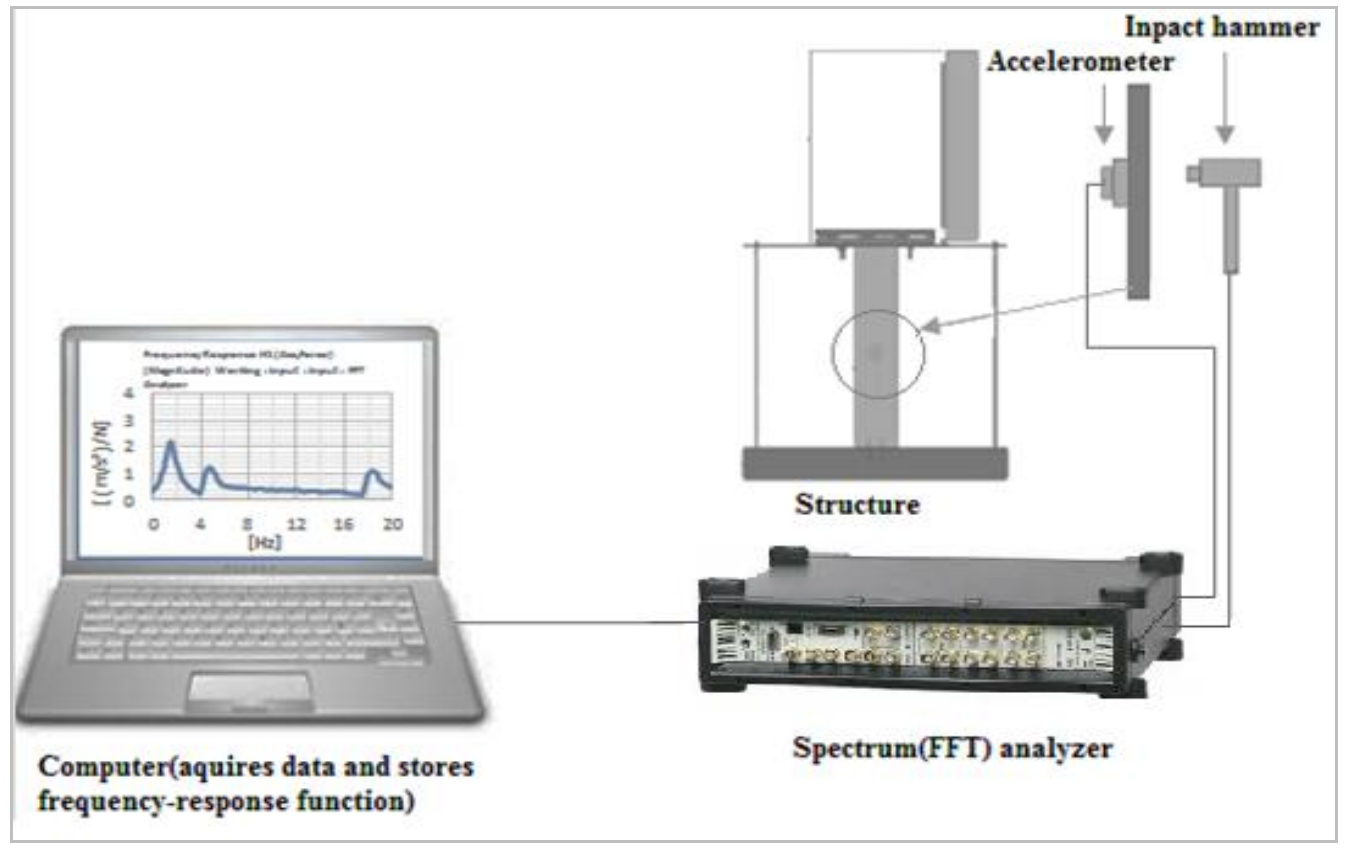

Figure 2 Schematic of hardware installation on structure.

The EMA is performed to obtain frequency responses of the system. From this frequency response modal characteristics are determined. Figure 3 shows frequency responses obtained.
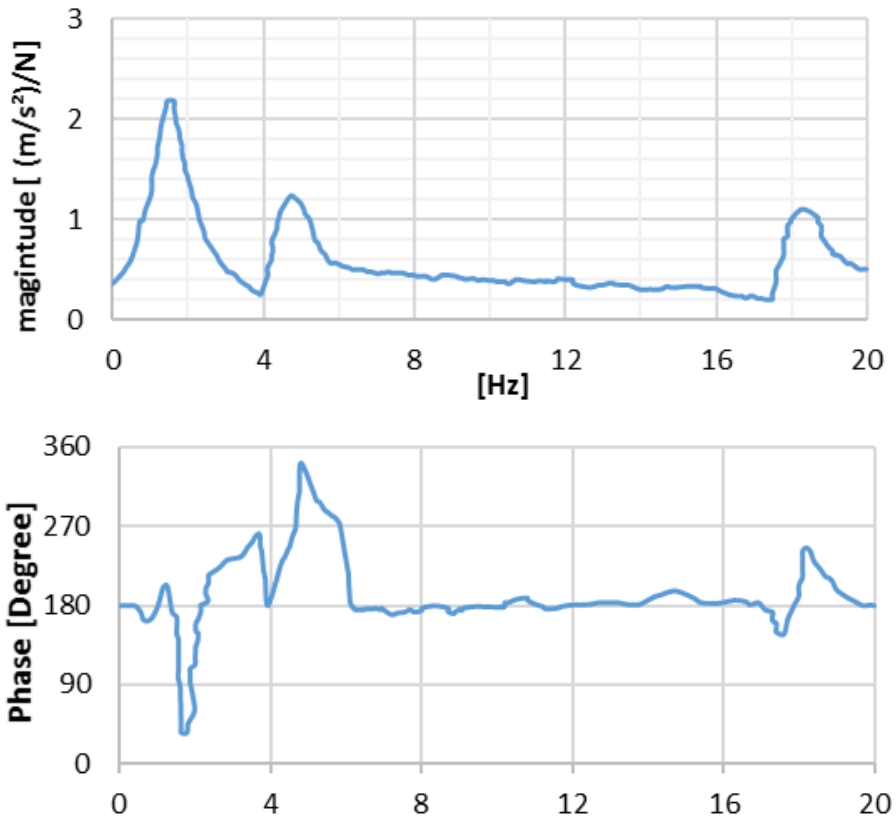

[Hz]

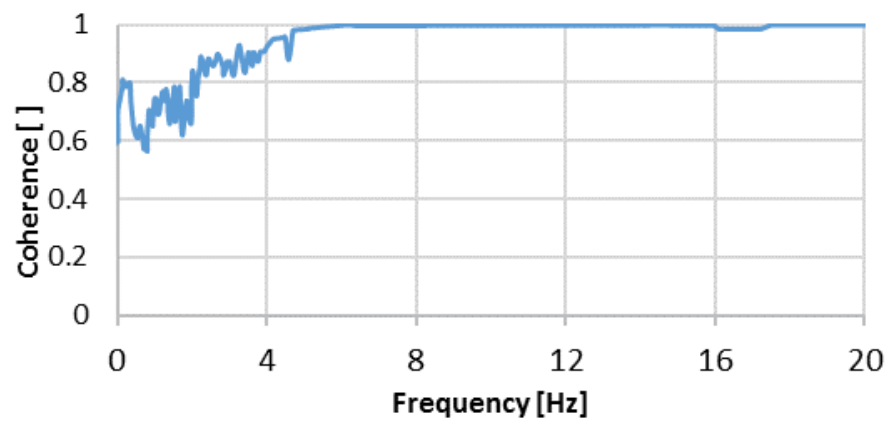

Fig. 3 Frequency response function (magnitude, phase and coherence). 
Second, the finite element model was developed for the experimental setup using FEMAP as a pre/post processor, the solver was NX Nastran 11. A normal modes analysis was performed to verify the natural frequencies and mode shapes. The bending and torsional modes are shown in the Figure 4.

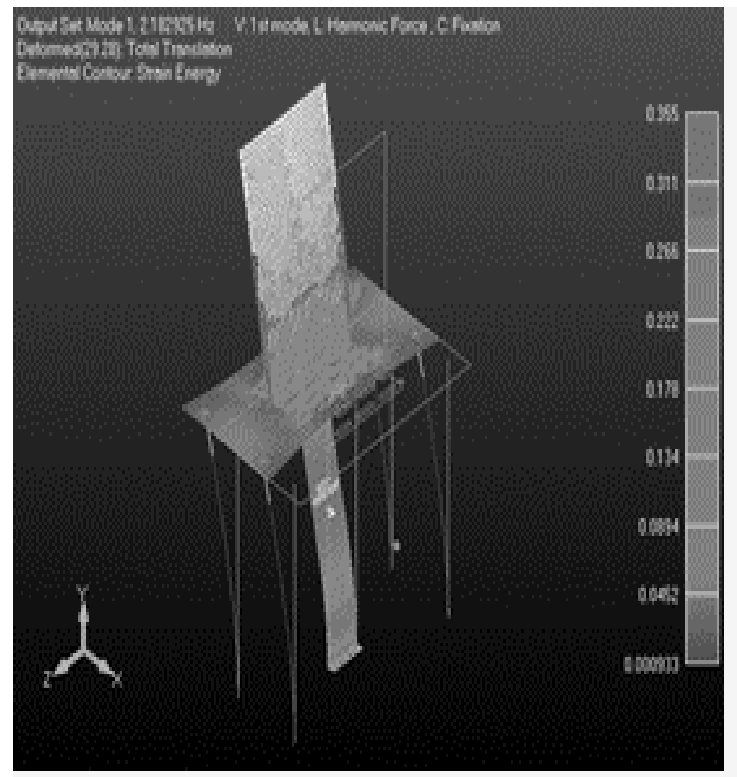

Bending mode

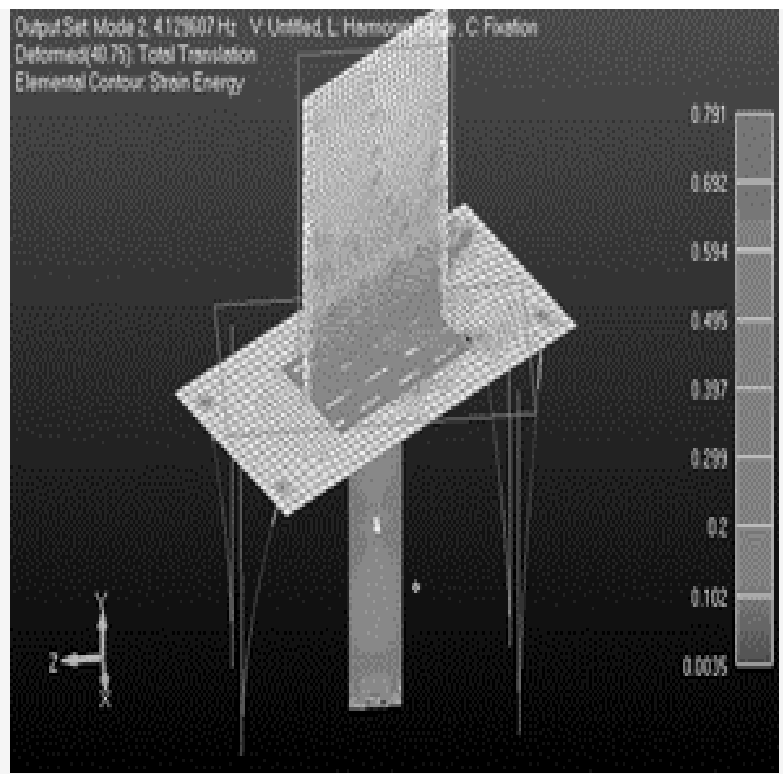

Torsional mode

Figure 4 showing that the bending and torsional modes

The force-time of the excitation hammer recorded from the experiment were analyzed using Fast Fourier Transform (FFT) to determine the frequency content of the signal, the frequency dependent load values are fed into the FE model for a frequency dependent excitation load. The frequency response is shown in Figure 5.
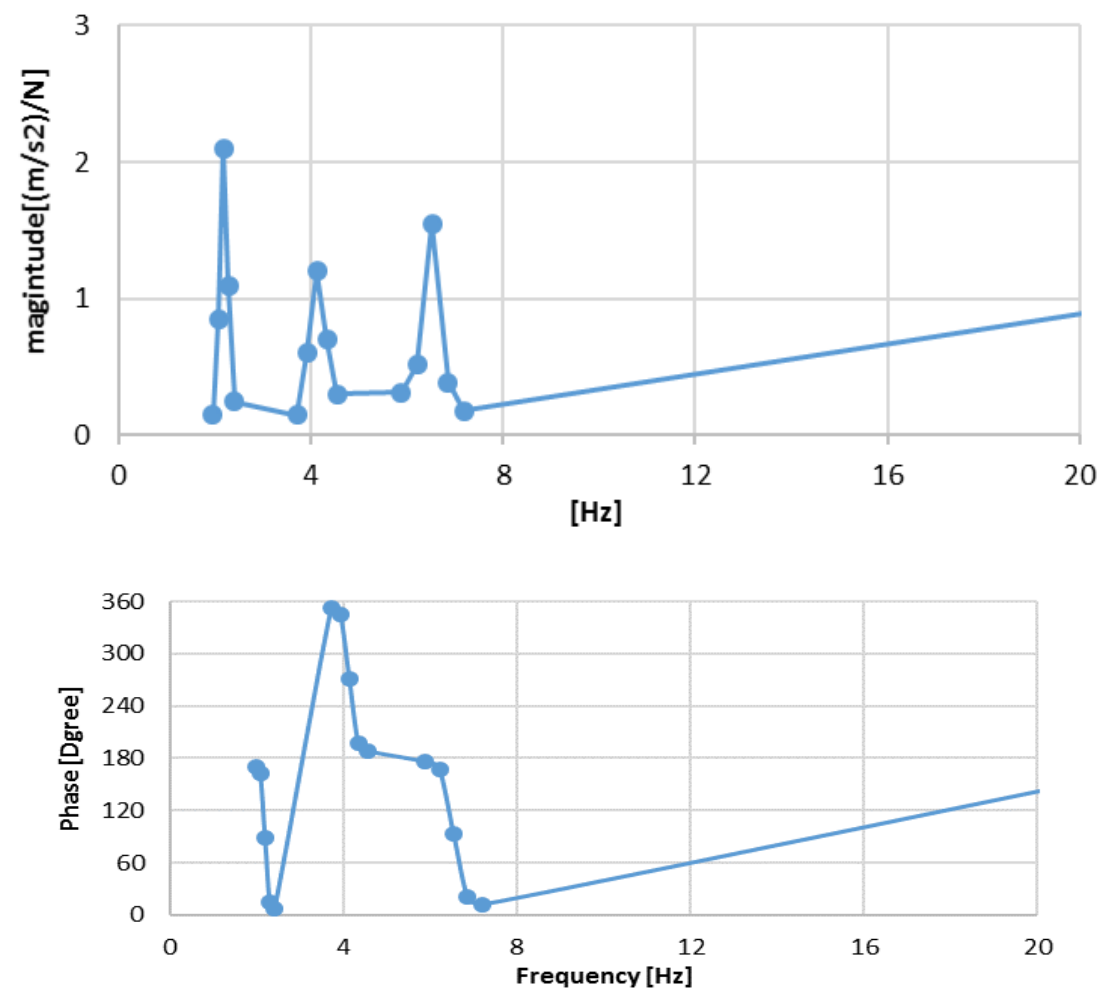

Fig. 5 Frequency response (magnitude, phase) of the flexible mount system and wing model identified with FEM for Bending and torsional modes. 
Table1 showing that the natural frequencies of the flexible mount system and wing model identified with the experimental modal analysis (EMA) and finite element model analysis.

Table1 Natural frequencies of the flexible mount system and wing model identified with the EMA\& FEM.

\begin{tabular}{|c|c|c|c|}
\hline Mode & EMA $(\mathrm{Hz})$ & FEM $(\mathrm{Hz})$ & Description \\
\hline 1 & 1.6 & 2.18 & First bending Frequency \\
\hline 2 & 4.8 & 4.129 & First torsional Frequency \\
\hline
\end{tabular}

\section{WIND TUNNEL TESTS}

The system is also tested in wind tunnel. These tests were performed in the wind tunnel of Aerodynamic Laboratory. This wind tunnel has a section test of approximately $0.25 \mathrm{~m}^{2}$ and maximum velocity of 30 $\mathrm{m} / \mathrm{s}$. Figure 6 shows the system mounted in the test section.

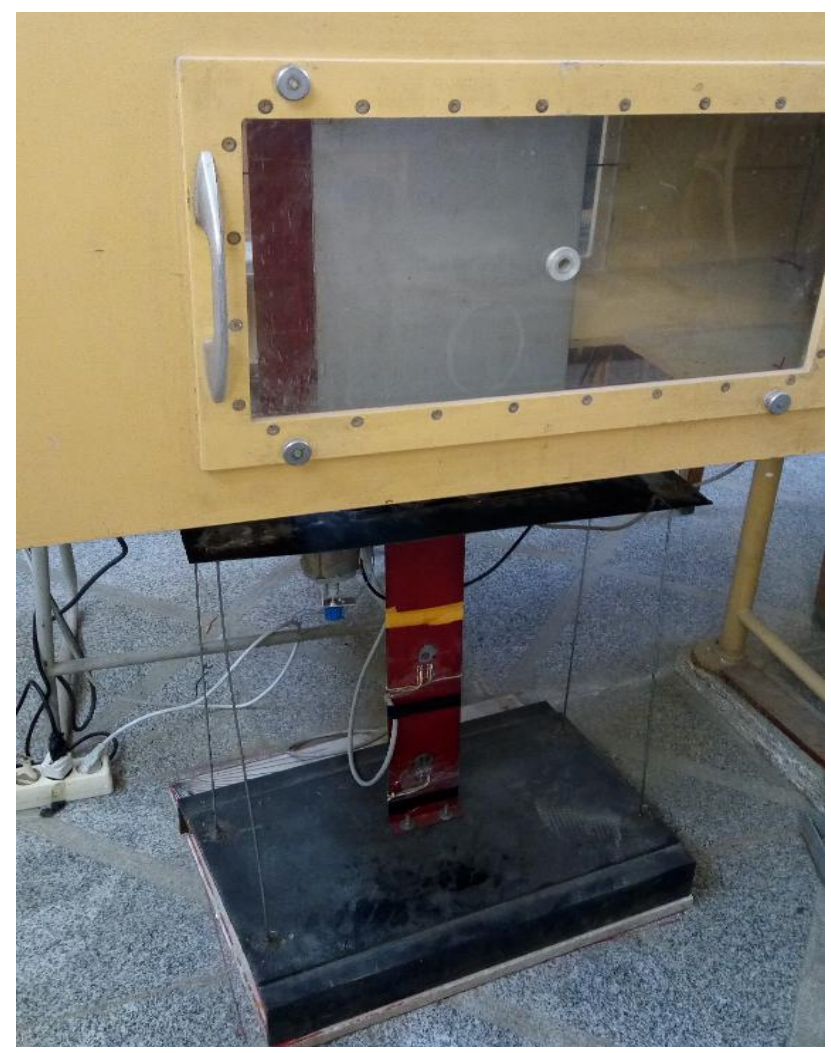

Fig. 6 Experimental system in the wind tunnel

The experimental system instrumentation for wind tunnel tests includes strain gauge bridges and a linear variable resistance. The position of the sensors in the experimental setup is shown in Figure 7.

Strain gauges are located in the center-line of the flat-plate strut in a maximum strain position. One strain gauge (KYOWA KFG-10-120-C1-11) is used to measure plunge displacements and the other (TML FCA-5-11) is used to measure pitch angles.

The wind tunnel velocity is obtained from the pressure measurements performed with a static Pitot tube manometer. 


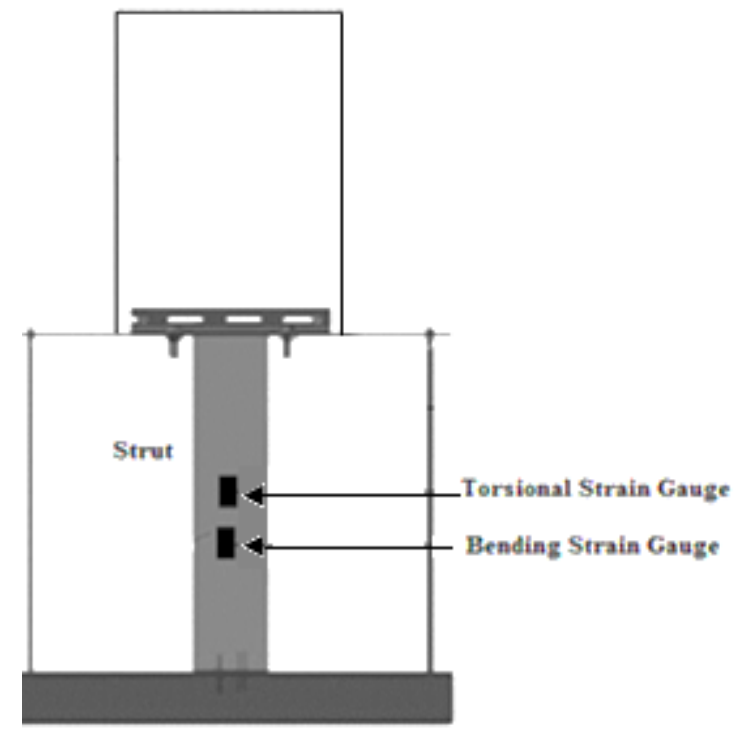

Figure 7 Position of the sensors for measurements during wind tunnel tests.

\section{MATHEMATICAL MODEL}

A mathematical model is an important tool to the theoretical development and posterior practical implementation of control systems. Analytical models are required for the development of simulations before any experimental implementation of controllers. In aeroelastic systems this fact is more relevant because any controller failure can result in destruction of the wind tunnel model and/or damage to the wind tunnel.

For the development of the aeroelastic model, the structural configuration of Figure 8 is used to represent the experimental setup. As can be seen, the structural model is divided into two parts, namely, the wing and the trailing edge flap.

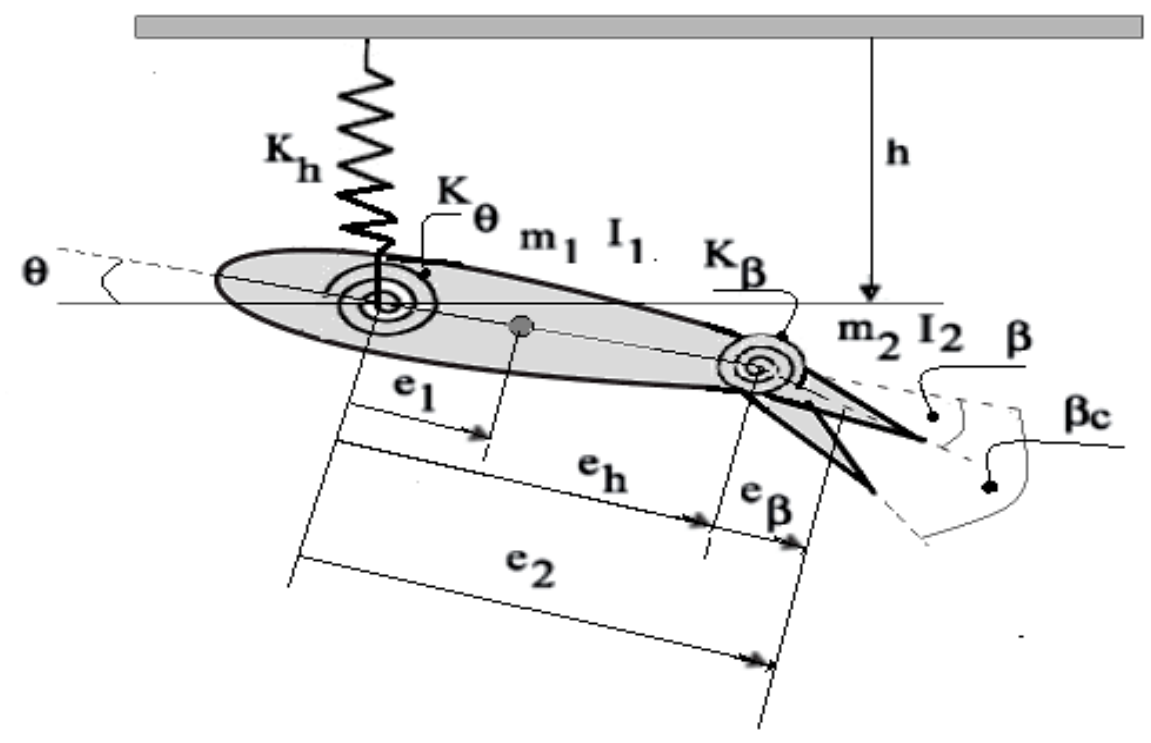

Fig. 8 structure representation of the physical model.

First, the potential energy of a rigid body consists of two terms, the strain energy of the spring and the gravitational potential energy is derived. These expressions are given by

$U_{\theta}=\frac{1}{2} k_{h} h^{2}+\frac{1}{2} k_{\theta} \theta^{2}+\frac{1}{2} k_{\beta}\left(\beta-\beta_{c}\right)^{2}$

$U_{g}=-m_{1} g\left[\mathrm{~h}+e_{1} \sin \theta\right] \cos \theta_{T}-m_{2} g\left[\mathrm{~h}+e_{2} \sin \theta+e_{\beta} \sin \beta\right] \cos \theta_{T}$

The expression for the kinetic energy obtained using the variables defined in Figure 6 is given by 
$T=\frac{1}{2} m_{1}\left[\dot{h}+e_{1} \dot{\theta}\right]^{2}+\frac{1}{2} I_{1} \dot{\theta}^{2}+\frac{1}{2} m_{2}\left[\dot{h}+e_{2} \dot{\theta}+e_{\beta} \dot{\beta}\right]^{2}+\frac{1}{2} I_{2}[\dot{\theta}+\dot{\beta}]^{2}$

Substitute in Lagrange's equations to get the equations of motion. Lagrange's equation can be expressed as in Equation 4, where $q_{i}$ the generalized are coordinates [14].

$\frac{d}{d t}\left(\frac{\partial T}{\partial \dot{q}_{i}}\right)-\frac{\partial K}{\partial q_{i}}+\frac{\partial U}{\partial q_{i}}=Q_{i}$

After applying the expressions for the kinetic and potential energies to Equation 4 results in the following system of equations. Assuming that the control surface stiffness is very large [11], [14].

$\left[\begin{array}{ll}m & S_{h \theta} \\ S_{h \theta} & I_{\theta}\end{array}\right]\left[\begin{array}{l}\bar{h} \\ \ddot{\theta}\end{array}\right]+\left[\begin{array}{cc}k_{h} & 0 \\ 0 & k_{\theta}\end{array}\right]\left[\begin{array}{l}h \\ \theta\end{array}\right]=-\left[\begin{array}{l}S_{h \beta} \\ S_{\theta \beta}\end{array}\right] \dot{\beta}+\left[\begin{array}{c}m \\ S_{h \theta}\end{array}\right] g \cos \theta_{T}+\left[\begin{array}{l}Q_{h} \\ Q_{\theta}\end{array}\right]$

Where;

$$
\begin{aligned}
& m=m_{1}+m_{2} \\
& \text { \& } I_{\theta}=m_{1} e_{1}^{2}+m_{2} e_{2}^{2}+I_{1}+I_{2} \\
& S_{h \theta}=m_{1} e_{1}+m_{2} e_{2} \\
& \text { \& } S_{h \beta}=m_{2} e_{\beta} \\
& \text { \& } S_{\theta \beta}=m_{2} e_{2} e_{\beta}+I_{2}
\end{aligned}
$$

The determination of expressions for the generalized forces $\boldsymbol{Q}_{\boldsymbol{h}}$ and $\boldsymbol{Q}_{\theta}$ are necessary to obtain the complete set of equations of motions. The generalized forces include non-conservative forces (damping) and external forces (aerodynamic). Structural damping is often characterized as a viscous force. The nonconservative forces can be presented as in Equation 6 if small motions of the experimental system are considered.

$\left[\begin{array}{l}Q_{h}^{d} \\ Q_{\theta}^{d}\end{array}\right]=-\left[\begin{array}{cc}m & S_{h \theta} \\ S_{h \theta} & I_{\theta}\end{array}\right]\left[\begin{array}{cc}\xi_{h} \omega_{h} & 0 \\ 0 & \xi_{\theta} \omega_{\theta}\end{array}\right]\left[\begin{array}{l}\dot{h} \\ \dot{\theta}\end{array}\right]$

The external forces are aerodynamic forces that result from the pressure distribution over the surface of the wing. These forces can be determined by using the Virtual Work Principle. A common method of approximating the aerodynamic forces is to use stability and control derivatives. The aerodynamic forces are represented as a linear function of angle-of-attack and control surface deflection and their rates as shown in Equations 7 and 8 [15].

$L=q S C_{L}=q S\left[C_{L_{0}}+C_{L_{\alpha}} \alpha+C_{L_{\beta} \beta} \beta+\frac{\bar{c}}{2 U_{0}}\left(C_{L_{\alpha}^{\circ}} \dot{\alpha}+C_{L_{q}} \dot{\theta}+C_{L_{\dot{\beta}}^{\prime}} \dot{\beta}\right)\right]$

$M=q S \bar{c} C_{L}=q S \bar{c}\left[C_{M_{0}}+C_{M_{\alpha}} \alpha+C_{M_{\beta} \beta} \beta+\frac{\bar{c}}{2 U_{0}}\left(C_{M_{\alpha}^{\prime}} \dot{\alpha}+C_{M_{q}} \dot{\theta}+C_{M_{\dot{\beta}}} \dot{\beta}\right)\right]$

The angle of attack at any chord-wise point $x$ at any time $t$ can be represented as in Equation 9 .

$\alpha(x, t)=\theta_{T}+\theta(t)+\frac{\dot{h}(t)}{U_{0}}+\frac{l(x) \dot{\theta}(t)}{U_{0}}-\frac{w_{g}(x, y, t)}{U_{0}}$

Where $\boldsymbol{\theta}_{T}$ is the initial angle of attack of the basis of the flexible system, $\boldsymbol{l}(\boldsymbol{x})$ is the chord-wise distance from the origin of the body fixed coordinate system to the point where the angle of attack is referenced (positive aft), $\boldsymbol{w}_{g}$ is the normal perturbation velocity of the local flow field (positive down relative to the free stream flow), and $\boldsymbol{U}_{\mathbf{0}}$ is the free-stream velocity. So, the external forces can be represented as in Equation 10 [11], [14],[15]. 


$$
\begin{aligned}
& {\left[\begin{array}{l}
Q_{h}^{e} \\
Q_{\theta}^{e}
\end{array}\right]=q S\left[\begin{array}{l}
-C_{L_{0}} \\
\bar{c} C_{M_{0}}
\end{array}\right]+q S\left[\begin{array}{l}
-C_{L_{\alpha \alpha}} \\
\bar{c} C_{M_{\alpha}}
\end{array}\right] \theta_{T}+\frac{q S \bar{c}}{2 U_{0}^{2}}\left[\begin{array}{ll}
-C_{L_{\alpha \alpha}^{\circ}} & -l(x) C_{L_{\dot{\alpha}}^{\circ}} \\
\bar{c} C_{M_{\dot{\alpha}}^{\circ}} & \bar{c} l(x) C_{M_{\alpha}^{\circ}}
\end{array}\right]\left[\begin{array}{l}
\bar{h} \\
\bar{\theta}
\end{array}\right]}
\end{aligned}
$$

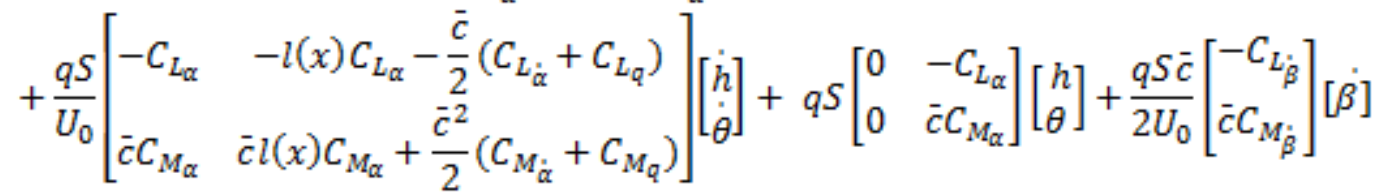

$$
\begin{aligned}
& +q S\left[\begin{array}{l}
-C_{L_{\beta}} \\
\bar{c} C_{M_{\beta}}
\end{array}\right][\beta]-\frac{q S}{U_{0}}\left[\begin{array}{ll}
-\frac{\bar{c}}{2 U_{0}} C_{L_{\alpha \bar{\alpha}}^{\circ}} & -C_{L_{\alpha \alpha}} \\
-\frac{\bar{c}}{2 U_{0}} C_{M_{\alpha \bar{\alpha}}} & \bar{c} C_{M_{\alpha \alpha}}
\end{array}\right]\left[\begin{array}{l}
w_{g} \\
w_{g}
\end{array}\right]
\end{aligned}
$$

The equation of motion is obtained when Equation 6 and Equation 10 are combined with Equation 5 resulting in Equation 11.

$$
\begin{aligned}
\left(M_{s}-M_{a}\right) \bar{\Psi}+ & \left(D_{s}-D_{a}\right) \dot{\Psi}+\left(K_{s}-K_{a}\right) \Psi \\
& =Q_{0}^{s}+Q_{T} \theta_{T}+M_{g} g \cos \theta_{T}+B_{2} \ddot{\beta}+B_{1} \dot{\beta}+B_{0} \beta+E W
\end{aligned}
$$

Where;

$$
\begin{aligned}
& \Psi=\left[\begin{array}{l}
h \\
\theta
\end{array}\right] \\
& w=\left[\begin{array}{l}
\dot{w_{g}} \\
w_{g}
\end{array}\right] \\
& M_{s}=\left[\begin{array}{ll}
m & S_{h \theta} \\
S_{h \theta} & I_{\theta}
\end{array}\right] \\
& M_{\alpha}=\frac{q S \bar{c}}{2 U_{0}^{2}}\left[\begin{array}{ll}
-C_{L_{\dot{\alpha}}} & -l(x) C_{L_{\dot{\alpha}}} \\
\bar{c} C_{M_{\dot{\alpha}}} & \bar{c} l(x) C_{M_{\dot{\alpha}}^{\dot{\alpha}}}
\end{array}\right] \quad D_{s}=-M_{s}\left[\begin{array}{cc}
2 \xi_{h} \omega_{h} & 0 \\
0 & 2 \xi_{\theta} \omega_{\theta}
\end{array}\right] \quad K_{s}=\left[\begin{array}{cc}
k_{h} & 0 \\
0 & k_{\theta}
\end{array}\right] \\
& D_{\alpha}=\frac{q S}{2 U_{0}}\left[\begin{array}{cc}
-C_{L_{\alpha \alpha}} & -l(x) C_{L_{\alpha \alpha}}-\frac{\bar{c}}{2}\left(C_{L_{\alpha}^{0}}+C_{L_{q}}\right) \\
\bar{c} C_{M_{\alpha}} & \bar{c} l(x) C_{M_{\alpha}}+\frac{\bar{c}^{2}}{2}\left(C_{M_{\dot{\alpha}}}+C_{M_{q}}\right)
\end{array}\right] \quad E=\frac{q S}{U_{0}}\left[\begin{array}{cc}
-\frac{\bar{c}}{2 U_{0}} C_{L_{\alpha}} & -C_{L_{\alpha \alpha}} \\
-\frac{\bar{c}}{2 U_{0}} C_{M_{\dot{\alpha}}} & \bar{c} C_{M_{\alpha \alpha}}
\end{array}\right] \\
& K_{\alpha}=q S\left[\begin{array}{ll}
0 & -C_{L_{\alpha \alpha}} \\
0 & \bar{c} C_{M_{\alpha}}
\end{array}\right] \\
& Q_{0}^{e}=q S\left[\begin{array}{l}
-C_{L_{0}} \\
\bar{c} C_{M_{0}}
\end{array}\right] \\
& Q_{T}=q S\left[\begin{array}{l}
-C_{L_{\alpha \alpha}} \\
\bar{c} C_{M_{\alpha}}
\end{array}\right] \\
& M_{g}=\left[\begin{array}{c}
m \\
S_{h \theta}
\end{array}\right] \quad B_{2}=\left[\begin{array}{c}
S_{h \beta} \\
S_{\theta \beta}
\end{array}\right] \\
& B_{1}=\frac{q S \bar{c}}{2 U_{0}}\left[\begin{array}{c}
-C_{L_{\beta}} \\
\bar{c} C_{M_{\beta}^{s}}
\end{array}\right] \\
& B_{0}=q S\left[\begin{array}{l}
-C_{L_{\beta}} \\
\bar{c} C_{M_{\beta}}
\end{array}\right]
\end{aligned}
$$

The static equilibrium of the system is related with the terms that are constant in Equation 11. This condition can be obtained by setting the time derivatives of Equation 11 to zero and solving for the generalized coordinates, leading to Equation 12.

$\Psi_{0}=\left(K_{s}-K_{a}\right)^{-1}\left[Q_{0}^{g}+Q_{T} \theta_{T}+M_{g} g \cos \theta_{T}+B_{0} \beta\right]$

The generalized coordinates and the control input can be represented in terms of a static $\Psi_{0}$ and a perturbation part $\stackrel{\sim}{\Psi}$, resulting in Equations 13 and 14 .

$$
\begin{aligned}
& \Psi=\Psi_{0}+\stackrel{\sim}{\Psi} \\
& \beta=\beta_{0}+\stackrel{\beta}{\beta}
\end{aligned}
$$

The equations above can be substituted in equation 12 resulting in the equation of motion for the system. 


$$
\begin{aligned}
& {\left[\begin{array}{c}
\tilde{\alpha} \\
\Psi \\
\dot{\alpha} \\
\Psi
\end{array}\right]=\left[\begin{array}{cc}
-\left(M_{s}-M_{\alpha}\right)^{-1}\left(D_{s}-D_{\alpha}\right) & -\left(M_{s}-M_{\alpha}\right)^{-1}\left(K_{s}-q S K_{\alpha}\right) \\
I & 0
\end{array}\right]\left[\begin{array}{c}
\dot{\alpha} \\
\Psi \\
\tilde{\Psi}
\end{array}\right]+\left[\begin{array}{c}
-\left(M_{s}-M_{\alpha}\right)^{-1} B_{2} \\
0
\end{array}\right] \begin{array}{c}
\tilde{\alpha} \\
\beta
\end{array}} \\
& +\left[\begin{array}{c}
\left(M_{s}-M_{a}\right)^{-1} B_{1} \\
0
\end{array}\right] \dot{\beta}+\left[\begin{array}{c}
\left(M_{s}-M_{a}\right)^{-1} B_{0} \\
0
\end{array}\right] \stackrel{\beta}{\beta} \\
& +\left[\begin{array}{c}
\left(M_{s}-M_{a}\right)^{-1} E \\
0
\end{array}\right] w
\end{aligned}
$$

\section{RESULTS}

The flutter speed was verified experimentally during wind tunnel experiments and compared with theoretical results.

The critical flutter velocity was verified in the first experimental test. The wind tunnel velocity was gradually increased and the pitch and plunge signals measured. The self-sustained oscillatory behavior of flutter was observed at velocity of $26.33 \mathrm{~m} / \mathrm{s}$. Figures 9,10 presents the plunge and pitch displacement measured during the experiments.

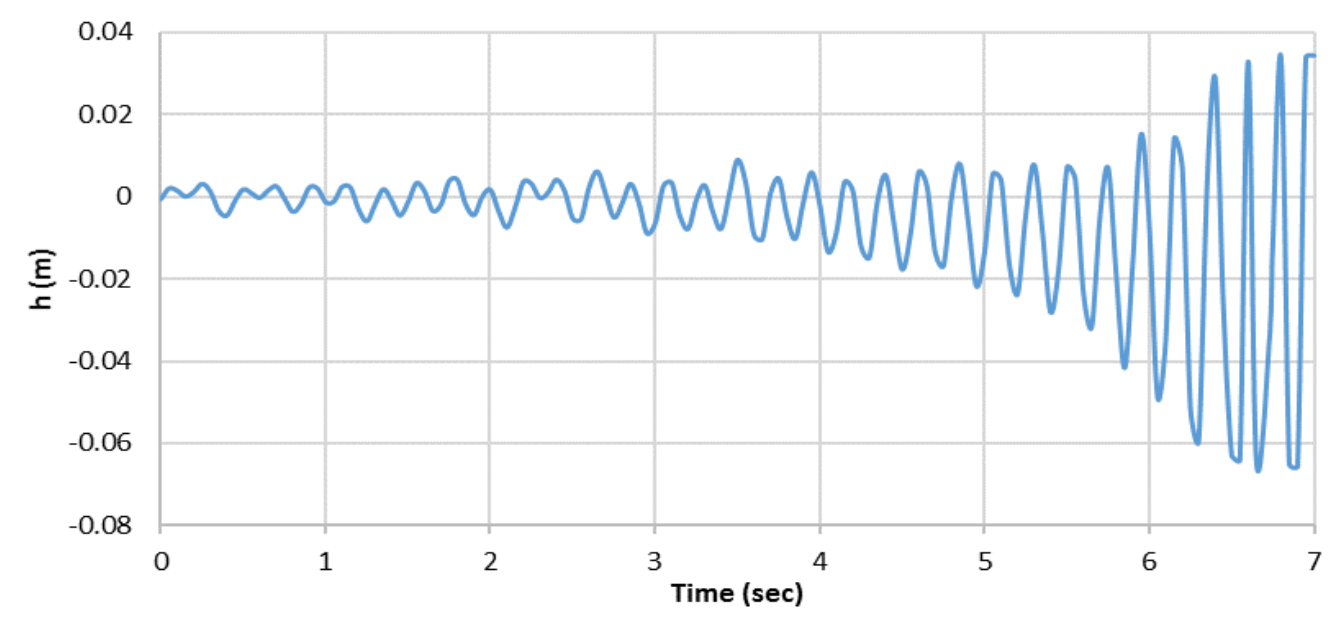

Fig. 9 Plunge response measured during wind tunnel tests at velocity of $26.33 \mathrm{~m} / \mathrm{s}$.

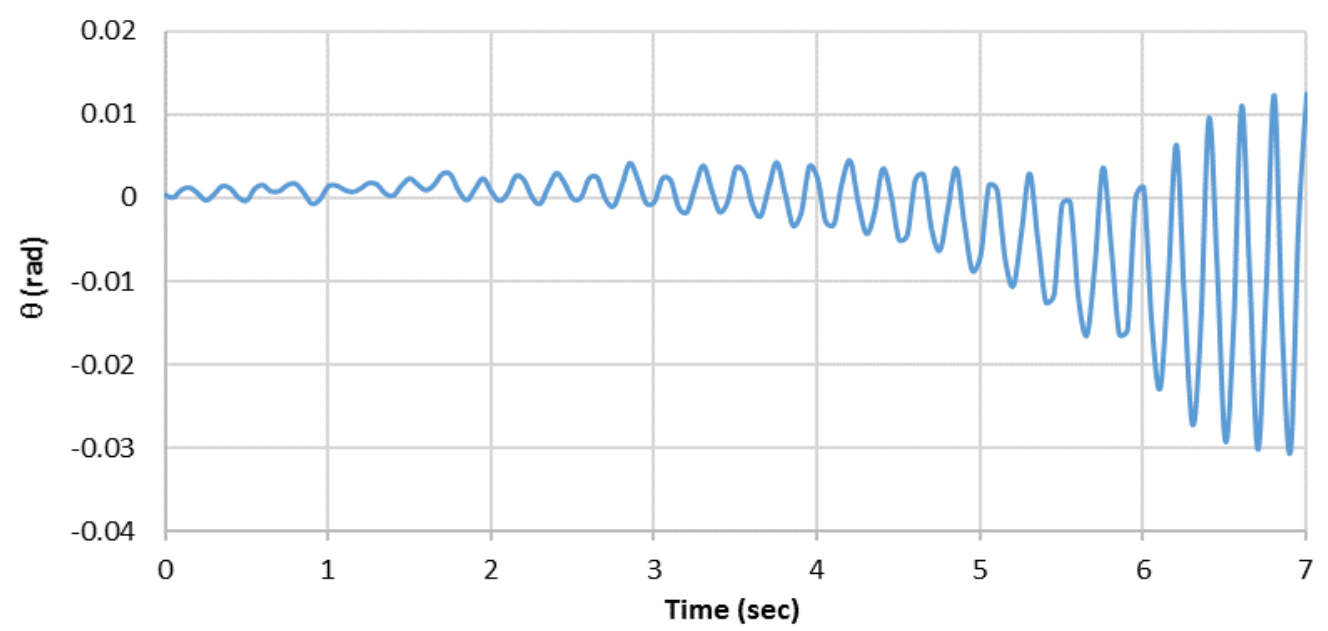

Fig. 10 Pitch angle measured during wind tunnel tests at velocity of $26.33 \mathrm{~m} / \mathrm{s}$.

Theoretically the flutter speed equal to $20.5 \mathrm{~m} / \mathrm{s}$ for steady flow according to $\mathrm{p}$ - method Figure $11,26.7 \mathrm{~m} / \mathrm{s}$ according to classical flutter Figure 12 and $27.8 \mathrm{~m} / \mathrm{s}$ according to k- method for unsteady flow as shown in the following Figure 13. 
VIBRATION ANALYSIS OF A FLAT PLATE UNDER AERODYNAMIC LOADING

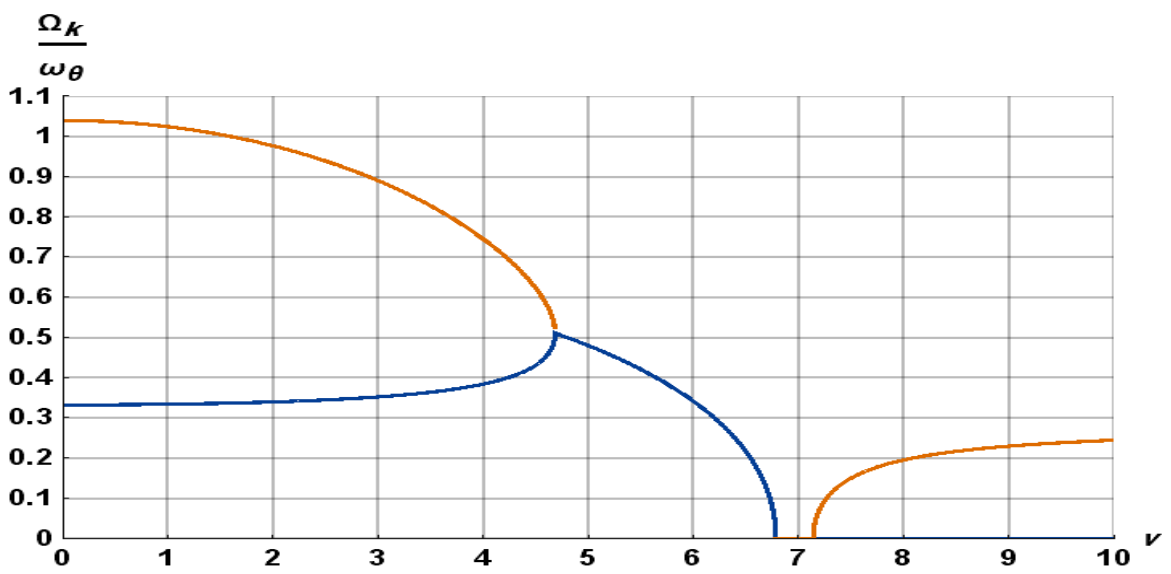

Fig. 11 Plot of the modal frequency $\left(\frac{n_{k}}{w_{g}}\right)$ versus to the reduced velocity

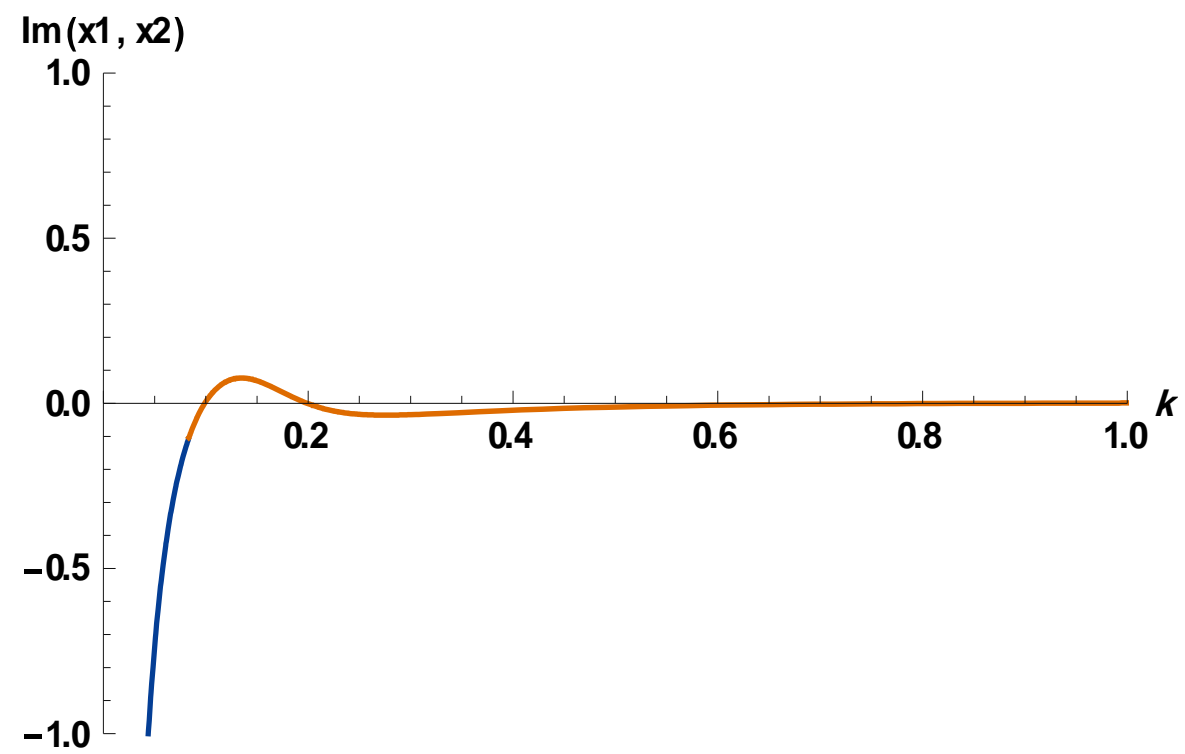

Figure 12 Imaginary parts of root versus to reduced frequency

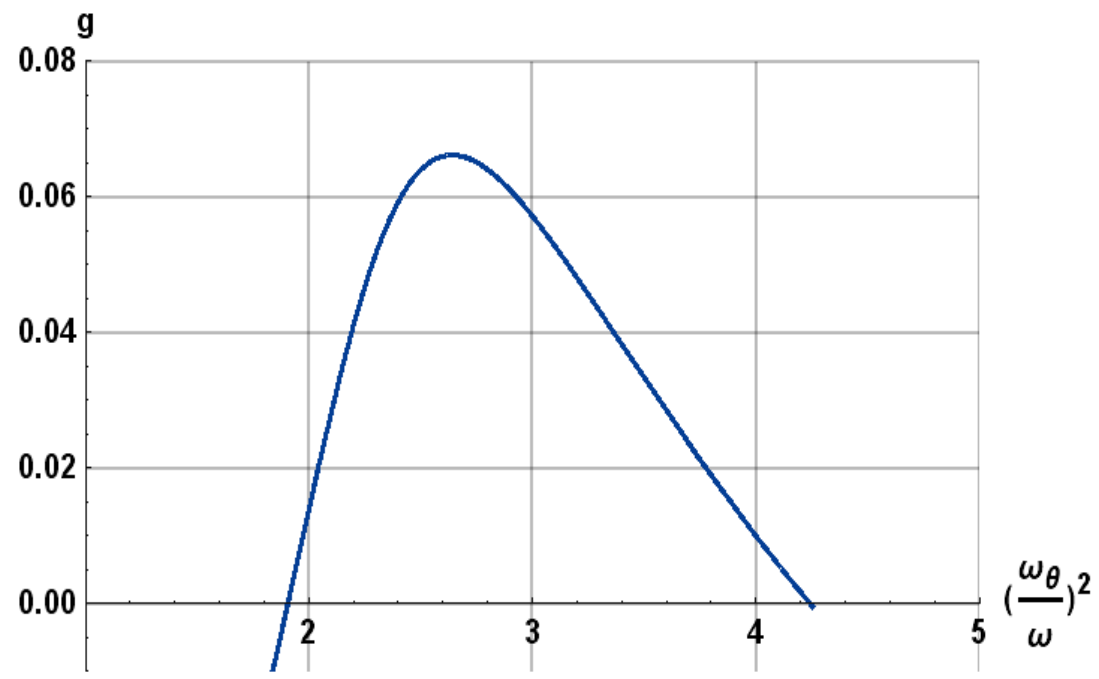

Figure 13 structural damping versus to real part of the frequency 
The numerical data used during the flutter experiments are presented in Table 2. These parameters are obtained from the experimental work and finite element model analysis.

Table 2 Numerical data for flutter experiments.

\begin{tabular}{|l|l|c|c|}
\hline \multicolumn{1}{|c|}{ symbol } & Description & Value & Unit \\
\hline$m$ & mass & 5.5 & $\mathrm{~kg}$ \\
\hline $\bar{c}$ & chord & 0.30 & $\mathrm{~m}$ \\
\hline$\omega_{h}$ & Plunge frequency & 9.42 & $\mathrm{rad} / \mathrm{s}$ \\
\hline$\omega_{\theta}$ & Pitch frequency & 28.26 & $\mathrm{rad} / \mathrm{s}$ \\
\hline \multicolumn{1}{|c|}{$S$} & area & 0.15 & $\mathrm{~m}^{2}$ \\
\hline$C_{L_{\alpha x}}$ & Coefficient of lift & $2 \pi$ & - \\
\hline
\end{tabular}

\section{CONCLUSIONS}

A flexible mount system was designed for flutter tests with rigid wings in a wind tunnel. A design procedure was developed to assure that classical flutter could be achieved during wind tunnel tests. This design procedure includes a finite element analysis complemented by experimental analysis methods.

A Finite Element model is only a design tool to the initial development of the system. As is verified in Tables 1, there are differences between frequencies of rigid body pitch and plunge modes determined by finite element and Experimental Modal Analysis/ERA analyses. These differences were expected since the finite element model is simplified and does not take into account some characteristics of the physical system. Also in the finite element model the connections of the rods and the flat plate strut are an ideal fixed-fixed end. This condition does not occur in the real model. Even with these simplifications the finite element model was an important tool for the design and verification of the flexible mount system.

The wind tunnel tests were performed to verify the evolution of rigid body modes with increasing velocity and also the flutter achievement. The frequency responses measured in these wind tunnel tests confirmed the expected behavior of the system, showing the evolution of modes and their coupling at flutter velocity. Time responses were also measured confirming the occurrence of flutter. The results obtained in this work prove that the flutter speed is the same for experimental and theoretical methods and occurs at $26.3 \mathrm{~m} / \mathrm{sec}$.

\section{REFERENCES}

[1] D. H. Hodges and G. A. Pierce, Introduction to Structural Dynamics and Aeroelasticity (Cambridge Aerospace Series, Vol. 15). 2011.

[2] V. Mukhopadhyay, "Historical Perspective on Analysis and Control of Aeroelastic Responses," J. Guid. Control. Dyn., vol. 26, no. 5, pp. 673-684, 2003.

[3] S. R. Cole, T. E. Noll, and B. Perry, "Transonic Dynamics Tunnel Aeroelastic Testing in Support of Aircraft Development," J. Aircr., vol. 40, no. 5, pp. 820-831, 2003.

[4] M. C. Sandford, I. Abel, and D. L. Gray, "Development and demonstration of a fluttersuppression system using active controls," Natl. Aeronaut. Sp. Adm., no. December 1975, 1975.

[5] B. Perry, III, S. R. Cole, and G. D. Miller, "Summary of an Active Flexible Wing program," J. Aircr., vol. 32, no. 1, pp. 10-15, 1995.

[6] M. Adams, "Design,Test,and Evaluation of Three Active Flutter Suppression Controllers," Natl. Aeronaut. Sp. Adm., 1992.

[7] M. Bennett and H. Durham, "Physical Properties of the Benchmark Models Program Supercritical Wing," Nasa Tech. Memo., 1993.

[8] M. R. Waszak, "odeling the Benchmark Active Control Technology Wind-Tunnel Model for Active Control Deesign Applications," NASA Cent. Aerosp. Inf., no. June, 1998.

[9] J. Ko, T. W. Strganac, and A. J. Kurdila, "Adaptive feedback linearization for the control 
of a typical wing section with structural nonlinearity," Nonlinear Dyn., vol. 18, no. 3, pp. 289-301, 1999.

[10] E. H. Dowell and D. Tang, "Nonlinear Aeroelasticity and Unsteady Aerodynamics," AIAA J., vol. 40, no. 9, pp. 1697-1707, 2002.

[11] C. De Marqui, E. M. Belo, and F. D. Marques, "A flutter suppression active controller," Proc. Inst. Mech. Eng. Part G-Journal Aerosp. Eng., vol. 219, pp. 19-33, 2005.

[12] Q. Wenmin, H. Rui, H. Haiyan, and Z. Yonghui, "Active flutter suppression of a multipleactuated-wing wind tunnel model," Chinese J. Aeronaut., vol. 27, no. 6, pp. 1451-1460, 2014.

[13] R. Huang, W. Qian, H. Hu, and Y. Zhao, "Design of active flutter suppression and windtunnel tests of a wing model involving a control delay," J. Fluids Struct., vol. 55, pp. 409427, 2015.

[14] C. De Marqui Jr., E. M. Belo, and F. D. Marques, "Wind-tunnel model and a controller for flutter suppression," Collect. Tech. Pap. - AIAA/ASME/ASCE/AHS/ASC Struct. Struct. Dyn. Mater. Conf., vol. 5, no. April, pp. 3663-3673, 2004.

[15] M. R. Waszak, "Modeling the Benchmark Active Control Technology Wind- Tunnel Model for Application To Flutter Suppression," AIAA Atmos. Flight Mech. Conf., no. 96, 1996.

\section{NOMENCLATURE}

\begin{tabular}{|c|c|c|}
\hline$B_{0}, B_{1}, B_{2}$ & wing semi-span, m & $e_{\beta}$ \\
\hline $\begin{array}{c}b \\
C_{L}, C_{M}\end{array}$ & $\begin{array}{l}\text { Semi-chord length , } \mathrm{m} \\
\text { aerodynamic lift and pitching } \\
\text { coefficients }\end{array}$ & \\
\hline$C_{L_{0}}$ & lift at zero angle of attack & $g_{h}, g_{\theta}$ \\
\hline$C_{M_{0}}$ & $\begin{array}{l}\text { pitching moment at zero angle of } \\
\text { attack }\end{array}$ & $\begin{array}{l}g \\
h\end{array}$ \\
\hline$C_{L_{\alpha x}}$ & lift curve slope & $I_{1}$ \\
\hline$C_{M_{\alpha}}$ & moment curve slope & \\
\hline$C_{L_{\beta}}$ & lift effectiveness & \\
\hline$C_{M_{\beta}}$ & Moment effectiveness & $I_{2}$ \\
\hline$C_{L_{\alpha}^{\prime}}$ & $\begin{array}{l}\text { plunge damping due to angle-of- } \\
\text { attack rate }\end{array}$ & \\
\hline$C_{M_{\alpha}^{\infty}}$ & $\begin{array}{l}\text { pitch damping due to angle-of- } \\
\text { attack rate }\end{array}$ & $K$ \\
\hline$C_{L_{q}}$ & plunge damping due to pitch rate & $k_{h}$ \\
\hline$C_{M_{q}}$ & pitch damping due to pitch rate & $k_{\theta}$ \\
\hline$C_{L_{\beta}^{\prime}}$ & rate lift effectiveness & $a$ \\
\hline$C_{M_{\beta}}$ & rate moment effectiveness & $l(x)$ \\
\hline $\bar{c}$ & mean aerodynamic chord & \\
\hline$D_{s}, D_{a}$ & $\begin{array}{l}\text { structural and aerodynamic } \\
\text { damping matrices }\end{array}$ & $L$ \\
\hline$e_{1}$ & $\begin{array}{l}\text { distance from origin of body fixed } \\
\text { reference axes to center of gravity } \\
\text { of system (excluding control } \\
\text { surfaces),m }\end{array}$ & $m_{1}$ \\
\hline$e_{2}$ & $\begin{array}{l}\text { distance from origin of body fixed } \\
\text { reference axes to center of trailing } \\
\text { edge control surface, } m\end{array}$ & $\begin{array}{l}M_{p}, M_{a} \\
M_{s}, M_{a} \\
M\end{array}$ \\
\hline$e_{h}$ & $\begin{array}{l}\text { distance from origin of body fixed } \\
\text { reference axes to hinge line of } \\
\text { trailing edge control surface, } m\end{array}$ & $\begin{array}{l}Q^{e} \\
Q^{d}\end{array}$ \\
\hline
\end{tabular}

distance from hinge line of trailing edge control surface to center of gravity of trailing edge control surface, $m$ structural damping gravitational acceleration, $\mathrm{m} / \mathrm{s}^{2}$ plunge deflection of the wing, $m$ Angular moment of inertia of the system (excluding control surfaces) about the origin of the body fixed reference axes, $\mathrm{kg} . \mathrm{m}^{2}$ angular moment of inertia of the trailing edge control surface about the origin of the body fixed reference axes, $\mathrm{kg} \mathrm{m} 2$ feedback matrix plunge stiffness , $\mathrm{N} / \mathrm{m}$ Pitch stiffness, $\mathrm{Nm} / \mathrm{rad}$ structural and aerodynamic stiffness matrices Chord-wise distance from the origin of the body fixed coordinate system to the point at which the angle of attack is referenced aerodynamic lift force, $\mathrm{N}$ mass of the system (excluding control surface), $\mathrm{kg}$ mass of the trailing edge control surface, $\mathrm{kg}$ aerodynamic pitching moment, $\mathrm{Nm}$ structural, aerodynamic and coupling mass matrices generalized external forces, $\mathrm{N}$ generalized non-conservative 
VIBRATION ANALYSIS OF A FLAT PLATE UNDER AERODYNAMIC LOADING

forces, $\mathrm{N}$

$Q_{0} \quad$ generalized aerodynamic force matrix at zero angle of attack

$Q_{T} \quad$ generalized aerodynamic force matrix associated with system angle

$q \quad$ dynamic pressure, $\mathrm{kg} / \mathrm{ms}^{2}$

$S \quad$ Planform area of the wing, $\mathrm{m}^{2}$

$T \quad$ kinetic energy, J

$t$ time, sec

$U_{g} \quad$ gravitational potential energy, $\mathrm{J}$

$U_{e} \quad$ elastic strain energy, $\mathrm{J}$
$U_{0} \quad$ freestream airspeed, $\mathrm{m} / \mathrm{s}$

$\alpha \quad$ The angle of attack, rad

$\beta_{c} \quad$ trailing edge control surface deflection, rad

$\omega_{h} \omega_{\theta} \quad$ modal frequencies, $\mathrm{rad} / \mathrm{s}$

$\theta$ pitch angle, rad

$\xi_{h}, \xi_{\theta} \quad$ modal structural damping ratio

$\rho_{\infty} \quad$ Density of the freestream air , $\mathrm{kg} / \mathrm{m}^{3}$

$\Gamma_{k} \quad$ modal damping

$\Omega_{k} \quad$ modal frequency 